\title{
Zugriffe von Strafverfolgungsbehörden auf Corona-Kontaktlisten
}

Nach den sogenannten Corona-Verordnungen vieler Bundesländer sind unter anderem Restaurants verpflichtet, zur Nachverfolgung möglicher Kontakte mit durch den Corona-Virus infizierten Personen Kontaktdaten ihrer Besucherinnen und Besucher zu erfassen. Zu den datenschutzrechtlichen Rahmenbedingungen dieser Datenerhebungen in Nordrhein-Westfalen informierte die LDI NRW am 25. September 2020.

In den Medien wurde zuletzt vermehrt darüber berichtet, dass die Polizei in anderen Bundesländern zur Aufklärung von Straftaten in mehreren Fällen auf diese Gästelisten zugegriffen habe. Zwischenzeitlich ist uns auch ein erster entsprechender Fall aus Nordrhein-Westfalen bekanntgeworden.

Die Zulässigkeit des Zugriffs auf die Corona-Kontaktlisten durch die Strafverfolgungsbehörden im Rahmen von strafrechtlichen Ermittlungsverfahren richtet sich insbesondere nach der Strafprozessordnung (StPO). Sobald die Polizei von einem Anfangsverdacht einer Straftat Kenntnis erlangt, ist sie nach dem Legalitätsgrundsatz verpflichtet, den Sachverhalt zu erforschen. Nach der sogenannten Ermittlungsgeneralklausel des $₫ 163$ StPO ist sie dabei zu Ermittlungen jeder Art befugt. Gleiches gilt für die Staatsanwaltschaften. Somit dürfen Polizei und Staatsanwaltschaft grundsätzlich sämtliche Ermittlungen durchführen, die aufgrund der Intensität ihres Grundrechtseingriffs nicht spezielle Eingriffsbefugnisse verlangen. Von einem derart gesteigerten Grundrechtseingriff kann im Fall des Zugriffs auf die Corona-Kontaktlisten regelmäßig nicht ausgegangen werden. Deshalb ist ein Zugriff auf Gästelisten auf Grundlage der Ermittlungsgeneralklausel grundsätzlich möglich.

Die Einsicht in die Corona-Kontaktlisten muss für die Ermittlungen jedoch auch erforderlich und verhältnismäßig sein. Das Vorliegen dieser Voraussetzungen muss von den Strafverfolgungsbehörden in jedem Einzelfall geprüft und festgestellt werden. Der Zugriff ist nur zulässig, wenn diese Voraussetzungen gegeben sind. Erforderlich und verhältnismäßig ist ein Zugriff auf die Corona-Listen in der Regel nicht zur Aufdeckung von Klein- kriminalität, sondern lediglich zur Verfolgung erheblicher Straftaten.

Neben der Einholung von Auskünften kommen auch besonders geregelte weitere Befugnisse der Strafverfolgungsbehörden in Betracht, so beispielsweise die Beschlagnahme eines Beweismittels oder die Anordnung einer Durchsuchung. Hierfür gelten jedoch spezielle Voraussetzungen.

Der Umstand, dass die Corona-Kontaktlisten schon kurz nach ihrer Einführung zur Strafverfolgung genutzt wurden, zeigt einmal mehr, dass dort, wo Daten erhoben werden, auch immer neue Begehrlichkeiten geweckt werden, diese Daten zu anderen Zwecken zu nutzen. Daher sollte in derartigen Fällen, in denen Daten zu einem besonderen Zweck erhoben werden, die Zweckbindung immer auch gesetzlich festgeschrieben werden.

Aus Sicht der Betroffenen kommt im Fall der Zugriffe auf Corona-Kontaktlisten erschwerend hinzu, dass die Möglichkeit der zweckändernden Verwendung zur Strafverfolgung vielfach zuvor nicht bekannt war. Dies schürt verständlicherweise Unmut, zumal wenn - wie in diesem Fall - zuvor durch öffentliche Stellen der Eindruck erweckt wurde, dass diese Daten ausschließlich für die Bekämpfung der Corona-Pandemie genutzt werden dürfen. Hieraus können Risiken für die Akzeptanz der Bevölkerung entstehen, einschneidende Maßnahmen, wie die im Zusammenhang mit der Corona-Pandemie, weiter hinzunehmen oder wahrheitsgemäße Angaben zu machen.

Vor dem Hintergrund, dass der LDI NRW für ganz NordrheinWestfalen erst ein Fall eines polizeilichen Zugriffes auf CoronaKontaktlisten bekannt geworden ist, gehen wir derzeit davon aus, dass die Strafverfolgungsbehörden hier mit dem erforderlichen Augenmaß vorgehen.

Helga Block, Landesbeauftragte für Datenschutz und Informationsfreiheit. Nordrhein-Westfalen 